\section{High-risk physics}

In the world of billion-dollar particle physics, one lab is taking an enormous gamble on its future. Geoff Brumfiel takes a look at Fermilab's hopes to host the next big machine.

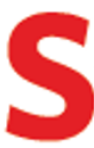
itting in a temporary office, Pier Oddone seems relaxed, even confident. Next month, the 60 -year-old physicist takes the helm of the leading highenergy physics laboratory in the United States, at a time when the lab's particle accelerator its raison dêtre - is scheduled to close within five years. But Oddone is optimistic about the lab's future. "It is a tremendous opportunity to move vigorously in new directions, ${ }^{n}$ he says.

Oddone will be the fifth director of the Fermi National Accelerator Laboratory, or Fermilab, a 2,100-person government facility located in Batavia on the edge of the Illinois prairie. The lab is home to the Tevatron - the most powerful particle collider in the world today. But within three years or so it will be supplanted by a bigger machine at CERN, the European high-energy physics laboratory near Geneva. When that happens, the US government plans to switch off the Tevatron, leaving Fermilab with a very uncertain future.

Fermilab's full-time employees are understandably feeling nervous, says Robert Roser, a physicist and spokesperson for the Collider Detector Facility (CDF), one of the Tevatron's detector experiments. "If it doesn't have any physics going on, a lab like this could be very tempting to shut down," he says bluntly.

\section{Making plans}

Oddone's hope is to make Fermilab the home to a newer, bigger accelerator called the International LinearCollider. This would be the largest collider yet: 30 kilometres long and capable of smashing electrons together at energies of 500 billion electronvolts $(\mathrm{eV})$ using state-of-the-art superconducting technology. It's so ambitious that physicists know it will take an international effort to build it. It would also cost a whopping US $\$ 6$ billion, according to more conservative estimates.

Oddone wants to work with international partners to begin building the linear collider at Fermilab by the end of the decade. To pull it off will require scientific cooperation, international diplomacy and a huge financial commitment from the US government.

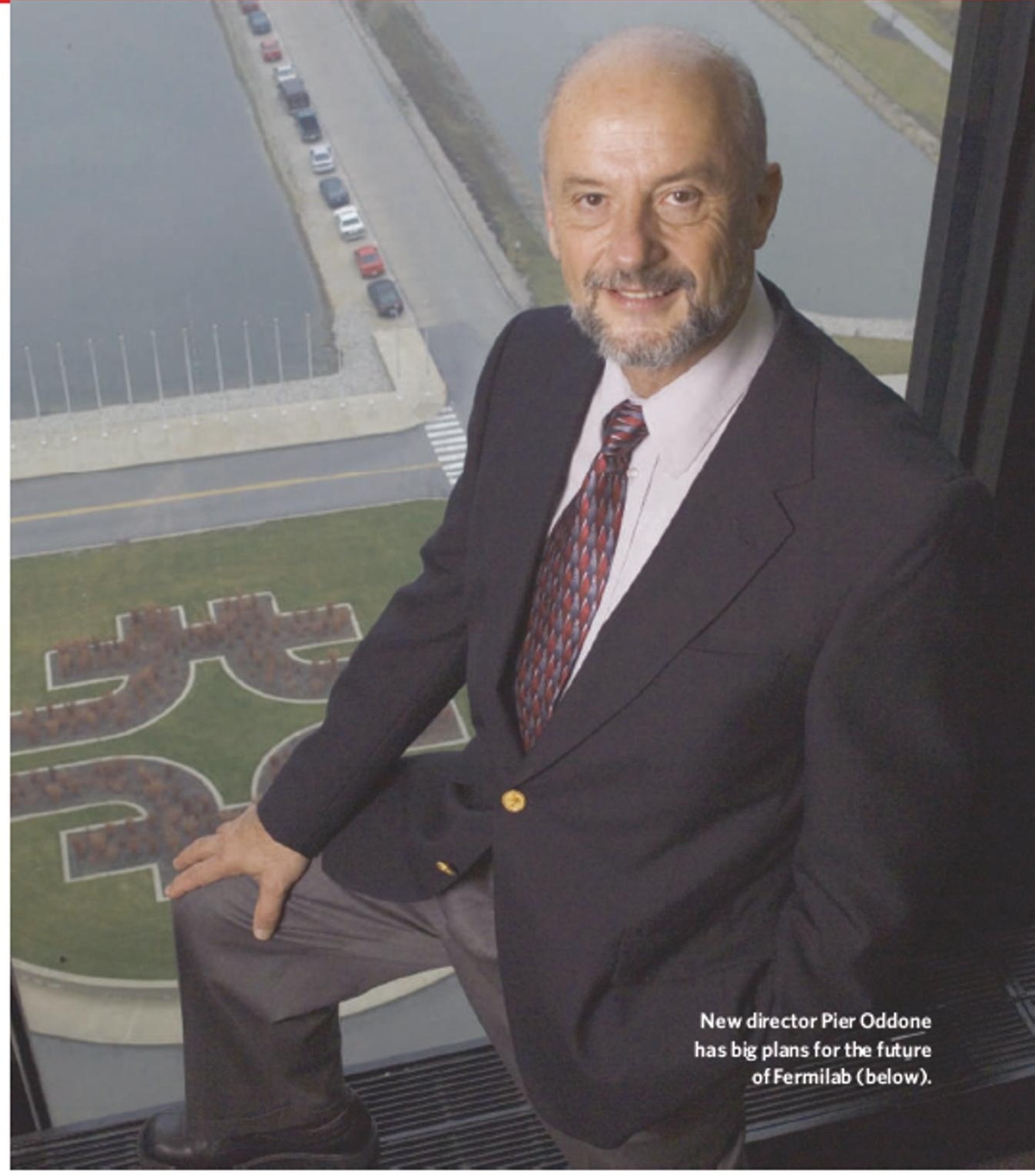

The plan is a gamble, one that could restore Fermilab to its former glory, or leave it adrift without a clear mission or future. Critics say that laboratory administrators are staking too much on a machine that has yet to win political support in the United States. But supporters say that to win big you have to dream big.

Physicists started building the Tevatron in 1979 with just such big dreams in mind -

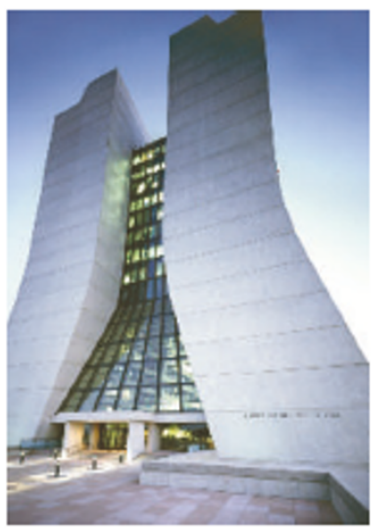

With further modifications, the collider netted the top quark in 1995. This discovery created elation among physicists and a headache for Fermilab management: what next?

Fermilab was not the only US particle-physics lab facing this dilemma. Nobel laureate Burton Richter, director emeritus at the Stanford Linear Accelerator Centre (SLAC) in Menlo Park, California, says he had a similar problem in 1992. The lab's electron accelamong them the goal of confirming the existence of the top quark. Quarks are the building blocks that make up protons, neutrons and other subatomic particles and the top and bottom quarks are the heaviest. The bottom quark had been detected at Fermilab in 1977, using the existing main ring accelerator. To find the heavier top quark meant smashing protons and anti-protons together at energies between 500 million and 1.5 billion $\mathrm{eV}$, which the Tevatron was designed to do. erator, which was the first to detect quarks in 1968 , was at the end of a long line of upgrades, and there was no space to expand.

So, Richter says, they decided to convert the accelerator into a powerful X-ray source, which could be used by biologists, chemists and materials scientists to determine the structure of molecules and materials. SLAC also began to diversify its research, moving into astrophysics, $\boldsymbol{\gamma}$-ray detection and cosmology. Today the lab is thriving and its budget is rising 
steadily. Just last month SLAC announced a major restructuring to complete its transition to a multipurpose facility. ${ }^{\alpha}$ The new structure is adapted to allow our scientists to get on with what they do best - make major discoveries," says SLAC director Jonathan Dorfan.

Rather than diversifying, Fermilab took the decision in the 1990 s to upgrade the Tevatron to even higher energies. This time their quarry was the Higgs boson, which, if theorists' predictions are correct, helps explain why everything in the Universe has mass. Bagging the Higgs would be a huge coup for Fermilab.

The US government spent hundreds of millions of dollars on the latest upgrade, which was completed in 2001. But the ageing Tevatron wasn't up to the task: its foundations had sagged over the years, and some of the new technology intended to boost its power behaved unpredictably. Budgets ran tight, and progress was slow. Today, after four difficult years, things are going smoothly, but so much time has been lost that few believe the Tevatron will discover the Higgs particle before s. CERN's machine is switched on in 2008.

Which leaves Fermilab in limbo, according to Richter. A year after CERN's machine turns on, the Tevatron will switch off, leaving lots of physicists with little to do, he says: "They have gotten themselves in a peculiar position, now the question is how do they get out of it?"

\section{Keeping the faith}

One solution may now be to diversify as SLAC has done. Fermilab has begun expanding its research in neutrinos - uncharged particles that pervade the Universe. Earlier this year, Fermilab turned on a $\$ 170$-million neutrino beam, aimed at a detector more than 700 kilometres away in the Soudan Mine in Minnesota. And several facilities are now being planned that would extend Fermilab's neutrino science well into the next decade.

But neutrino research will not be enough to sustain the facility at its present level, and many physicists at Fermilab remain disdainful of distractions from the lab's original mission. "Diversifying is the easier solution, but the hard road is more meaningful," says YoungKee Kim, a physicist and spokesperson for the CDF. "From my point of view, we have to have a high-energy centre in the United States, or we'll lose the energy frontier."

Oddone agrees. ${ }^{\alpha}$ The International Linear Collider is the greatest opportunity for this lab right now, he says. With the US Department of Energy, Fermilab is stepping up its accelerator and detector research to make a strong bid for the new machine. If all goes well, Oddone says, the lab will again be the centre of world physics by early next decade as the linear collider probes exotic particles, such as the Higgs, in greater detail.

But that's a big if. Collaborations on the
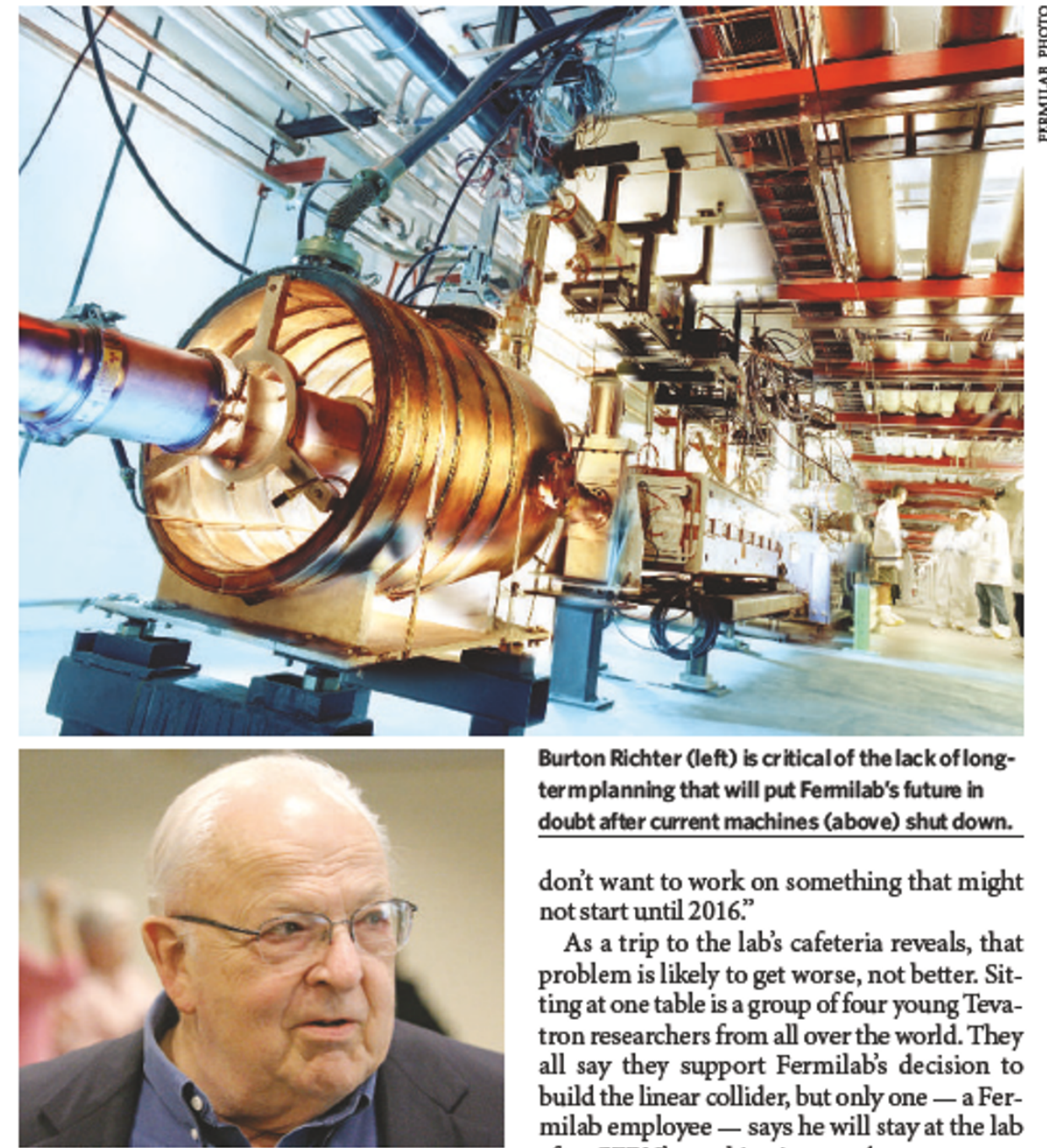

Burton Richter (left) is critical of the lack of longtermplanning that will put Fermilab's future in doubt after current machines (above) shut down.

don't want to work on something that might not start until 2016."

As a trip to the lab's cafeteria reveals, that problem is likely to get worse, not better. Sitting at one table is a group of four young Tevatron researchers from all over the world. They all say they support Fermilab's decision to build the linear collider, but only one - a Fermilab employee - says he will stay at the lab after CERN's machine is turned on.

The lab is doing its best to stem the tide. One scale needed to build the linear collider are notoriously difficult - fraught with cost overruns, infighting and international politics. And even if the collider does move forward, it may not be built in the United States. Japan is energetically pursuing its own plans to host the machine at its KEK facility, according to Yoji Totsuka, KEK's director-general.

The uncertainty is making it difficult for Fermilab to retain the thousands of visiting scientists it needs to run its existing experiments. Already, the Tevatron's two main detectors, CDF and DZero, are suffering from staff shortages. "A lot of people are in the process of moving or have already moved," says stopgap is a computing centre that will allow US researchers to monitor their experiments at CERN from a distance. "We're going to see the same data as they see in CERN," says Avi Yagil, who is heading the centre's development. Fermilab is a critical link on the Grid, a distributed computing network that will transmit the Large Hadron Collider's data to the international physics community.

Yagil says he hopes that these efforts can prevent the lab from becoming, as he puts it, scorched earth", but will it be enough to keep the lab going? Richter doesn't think so, and he is highly critical of the lab's leadership for not doing more to diversify their programme. ${ }^{\alpha}$ There has never been a coherent long-term view at Fermilab of what you're going to do in the next act, that is in 10 or 15 years," he says. "And that's what got them where they are."

Oddone begs to differ. Fermilab has the staff, knowledge and space to build the next big accelerator, he says. But anything less than a full commitment will not make the dream come true. "Yes, there are tremendous risks," he says. "But the questions we are trying to answer are also tremendous."

Geoff Brumfiel is Nature's Washington

physical sciences correspondent. really unique physics opportunity coming CERN in the next two or three years, and they
This situation also makes it harder to attract the expertise needed to develop linear collider types. "It's very difficult," says Hend Weerts, who has taken a sabbatical from Michigan State University in Lansing to work 